\title{
Ecosistemas locales de aprendizaje ante la globalización tecnológica. Retos de los modelos educativos digitales pospandemia
}

\section{Placing Local Learning Ecosystems at the Forefront of Technological Globalization: Challenges faced by Post-Pandemic Digital Educational Models}

\author{
Esperanza Román Mendoza \\ George Mason University (Estados Unidos) \\ eromanme@gmu.edu \\ Cristóbal Suárez-Guerrero \\ Universitat de València (España) \\ cristobal.suarez@uv.es
}

\section{RESUMEN}

La educación frente a la covid-19 ha puesto en práctica una infinidad de respuestas, todas urgentes, tentativas, generadas por ensayo y por error, y todavía pendientes de validación. Para comprender mejor el impacto de estas respuestas, el presente artículo defiende, partiendo de la teoría de los ecosistemas de aprendizaje, la necesidad de investigar cómo han influido las tecnologías de ámbito global en la construcción de experiencias locales de aprendizaje. Además, pone de manifiesto la necesidad de estudiar qué tipo de apoyo tanto teórico como práctico se requiere para reconducir los posibles efectos nocivos de la introducción de la tecnología de emergencia. A tal efecto, se describen las consecuencias de fenómenos globalizadores, como el tecnocentrismo, el solucionismo tecnológico, la plataformización y la economía de datos, proponiendo la reflexión, la concienciación para la transformación y la acción desde los ecosistemas locales de aprendizajes como vía imprescindible para la conceptualización de nuevos modelos educativos digitales pospandemia. El artículo también resume las aportaciones principales de los otros trabajos que forman parte de la monografía.

\section{PALABRAS CLAVE}

COVID-19; modelos educativos; educación digital; ecosistemas de aprendizaje; pedagogía crítica.

\section{ABSTRACT}

Education has offered multiple, urgent, and tentative responses to Covid-19, generated by trial and error and still pending validation. To understand the impact of these responses, this article advocates for the study of how global technologies have influenced the pursuit of local learning experiences. In addition, the article emphasizes the need to investigate what kinds of theoretical and practical support are required to counterbalance the possible negative effects of emergency technology responses. Accordingly, it describes the consequences of globalizing phenomena, such as technocentrism, technological solutionism, platformization and data economy, and suggests that the reflection, critical consciousness engaged in transformation and action from within each local learning ecosystem are necessary to conceptualize new post-pandemic educational digital models. The article also summarizes the main contributions of the works included in this special issue.

\section{KEYWORDS}

COVID-19; educational models; digital education; learning ecosystems; critical pedagogy. 


\section{CITA RECOMENDADA}

Román, E. \& Suárez-Guerrero (2021). Ecosistemas locales de aprendizaje ante la globalización tecnológica. Retos de los modelos educativos digitales pospandemia. RIITE Revista Interuniversitaria de Investigación en Tecnología Educativa, 11, 1-11. https://doi.org/10.6018/riite.503001

\section{INTRODUCCIÓN}

En enero de 2020, nadie se podía imaginar que los sistemas educativos del mundo estaban a punto de experimentar el mayor cambio de su historia. Semanas después del estallido de la alarma global provocada por el virus SARS-CoV-2, la mayoría de los centros de formación, a nivel mundial y en todos los contextos educativos, tuvieron que cerrar sus aulas y adaptarse a la imposibilidad de realizar su cometido de forma presencial. Ningún sistema educativo estaba preparado para este proceso inédito $y$, aunque todos ellos se enfrentaban a un mismo virus, las condiciones estructurales de cada país y la forma de gestión socioeducativa marcaron la diferencia (Organización de las Naciones Unidas [ONU], 2020; World Bank, 2020).

Para que la denominada educación digital de emergencia (Hodges et al., 2020) pudiera convertirse en una respuesta acertada al contexto de la pandemia, había que salvar, por lo menos, tres brechas: la relativa al acceso a la tecnología, la referida a su uso —ligada a la competencia digital- y la brecha escolar, relativa al soporte tecnopedagógico que el sistema y los docentes debían tener de cara a ese nuevo escenario educativo (Fernández Enguita, 2020). El proceso no consistió en sustituir una herramienta por otra, sino en mantener cierto grado de continuidad pedagógica en una situación de emergencia sanitaria de efectos completamente imprevisibles. Puesto que se partía de un desigual grado de aceptación e implementación de la tecnología en los procesos formales de enseñanza/aprendizaje, los resultados y las consecuencias de esta transición de emergencia a la enseñanza mediada por la tecnología fueron de lo más dispar, como queda reflejado en los informes publicados por la UNESCO durante la pandemia. Pero, entre toda esa inmensa variedad de experiencias y medidas emerge un tema recurrente y generalizado: ¿Cuáles van a ser las implicaciones pedagógicas, tanto positivas como negativas, del impacto que estas experiencias de emergencia han tenido entre las partes implicadas en el proceso educativo? En otras palabras, ¿cómo se puede encauzar la educación pospandemia a la vista de los procesos de transformación que han ocurrido de forma local y los condicionantes tecnopedagógicos que se han impuesto desde la globalidad?

Dada la importancia de dichas cuestiones, no resulta extraño que en los últimos meses hayamos sido testigos de una explosión de estudios y publicaciones académicas sobre los efectos de la pandemia en los sistemas educativos. Una rápida consulta en World of Science (WoS) nos muestra más de 25000 publicaciones relacionadas con los términos "pandemia" y "educación" entre los años 2019 y 2021. Sin embargo, aunque el español ocupa el segundo lugar en cuanto a número de publicaciones sobre la pandemia en todas las áreas del conocimiento, la diferencia con la cantidad de publicaciones en inglés es abrumadora: $483(1,93 \%)$ resultados en español frente a 24281 (97\%) en inglés. Este contraste no supone un fenómeno nuevo en el mundo académico, donde existe la común percepción de que el alcance de una publicación fuera del ámbito hispanohablante y, por consiguiente, la visibilidad de sus autores, es mayor si se publica en inglés que si se utiliza una lengua semiperiférica, como el español (Burgess, 2014; Hayland, 2012).

Sin embargo, si restringimos la búsqueda al área de investigación educativa, el panorama es algo más esperanzador para el español: 244 (8,3\%) publicaciones en español frente a 2500 $(85,4 \%)$ en inglés, de un total de 2925 indexadas bajo esa categoría en WoS a fecha de octubre de 2021. Podemos apuntar sin temor a equivocarnos que la comunidad que investiga temas relacionados con la educación es más consciente de las implicaciones epistemológicas que conlleva publicar en una lengua diferente de la que se utiliza para llevar a cabo la investigación y que impone modelos discursivos ajenos a la cultura de los autores. Otro dato alentador en este sentido es que el número de revistas en español indexadas bajo la categoría de educación es 
mucho mayor que en otros ámbitos de la ciencia, como la farmacología o las ingenierías, donde la preponderancia del inglés como lingua franca es un hecho indiscutible desde hace años. Como ejemplo de ello, se puede mencionar las 378 revistas en español de acceso abierto sobre educación indexadas en el Directorio de Revistas de Acceso Abierto (Directory of Open Access Directory) frente a las 1064 en inglés.

Todo lo anterior no significa necesariamente que resulte imposible o censurable formular una visión epistemológica en el campo de la educación nacida en la semiperiferia. Y esto es lo que se refleja en este número monográfico. La complejidad del tema elegido, los modelos educativos digitales, y la necesidad de proporcionar un contexto razonado, detallado y contrastado, que permita que las experiencias locales sirvan de base tanto para subsiguientes experiencias en el mismo ámbito así como para el desarrollo de modelos educativos de alcance global, ha tenido como consecuencia que los docentes-investigadores que han respondido a nuestro llamado presenten sus trabajos en la lengua en la que fueron concebidas $-y$ contextualizadas - sus investigaciones. Nuestra aportación, como editores invitados del monográfico, también va a servirse de nuestra lengua, que, aunque semiperiférica, o quizá precisamente por ello, es capaz de organizar el discurso tecnopedagógico desde una perspectiva diferente a la ofrecida en el mundo anglosajón. El idioma, así, forma parte de la conceptualización del objeto de estudio y de las propuestas que se derivan de su análisis. Usar un idioma semiperiférico contribuye, además, a la descolonización del campo de la tecnología educativa.

\section{MODELOS EDUCATIVOS DIGITALES: MÁS ALLÁ DE LA HERRAMIENTA}

La tecnología digital ofrece una amplia gama de posibilidades, no siempre exentas de mitos y de controversia en torno a su potencial para facilitar el aprendizaje en todos los niveles y contextos educativos (Ornellas \& Sancho, 2015). Sin embargo, debido a la celeridad con la que avanza la tecnología y a la prevalencia del uso de los recursos tecnológicos como meros instrumentos orientados a llevar a cabo una determinada tarea, una gran parte de los estudios sobre la integración de la tecnología en el ámbito educativo publicados hasta la fecha se concentran en los artefactos, minimizando cómo la tecnología digital forma parte articulada dentro de los modelos educativos en los que se integra (Castañeda \& Selwyn, 2018). Dado que ninguna tecnología es una entidad neutral que opera en un vacío contextual, cuando se habla de tecnologías que median una relación tan compleja y angular como es el aprendizaje en el marco de la educación formal, resulta necesario hacer significativa esa tecnología desde una lectura pedagógica que le brinde sentido, ubicación y arraigo dentro de un proyecto humano (SuárezGuerrero, 2014; Teräs et al., 2020). La tecnología es necesaria pero no suficiente en educación. Por ello, hay que enfocar la tecnología desde modelos educativos digitales que respondan a las características del ecosistema donde se implementan. Idear modelos educativos es hacer educación pensado desde la necesidad educativa y no solo desde la instrumentalidad tecnológica.

Sin embargo, durante la pandemia, la posibilidad de continuar la actividad educativa una vez interrumpida la presencialidad fue directamente proporcional a la capacidad que tuvieron las partes implicadas en ella para acceder y aprovechar la tecnología digital (Pulido-Montes \& Ancheta-Arrabal, 2021; Williamson et al., 2020). Es muy posible, entonces, que la pandemia haya tenido un doble efecto sobre la percepción del papel de la tecnología en la educación. Por una parte, nadie pone en duda que la educación digital de emergencia no se pudo planificar de la misma manera que la educación digital "tradicional" y que, por lo tanto, no se pueda extrapolar los juicios sobre su efectividad a otras situaciones no asociadas a la emergencia. Pero, por otra, a pesar de que se tenga claro que la educación digital de emergencia fue algo transitorio, es innegable que esta ha servido para abrir nuevos horizontes tecnopedagógicos más acordes con la pedagogía crítica, así como para todo lo contrario: perpetuar el tecnocentrismo que ya existía antes de la covid-19 (Selwyn et al., 2020). A continuación, se repasan los aspectos más característicos del tecnocentrismo y el solucionismo tecnológico para proceder después a describir cómo el concepto de ecosistema de aprendizaje, que subyace a la visión de modelo educativo digital, puede servir para contrarrestar los efectos predatorios que la tecnología puede tener sobre él. 
En primer lugar, los fracasos en la introducción de las tecnologías en el aula se atribuyen generalmente a una deficitaria formación en competencias digitales o al desigual acceso a la tecnología. Las empresas que desarrollan la tecnología y los medios de comunicación digital que se usan en la actualidad en educación a nivel mundial se han cuidado bien de que los patinazos tecnológicos en educación no se perciban como el resultado de la utilización de un patín defectuoso o inadecuado para deslizarse sobre ciertas superficies, sino que se imputan a la falta de pericia por parte de los patinadores para implementarlos (Cuban \& Jandrić, 2015). Así, se aboga por una mayor formación del profesorado y alumnado, pero en lugar de ofrecer oportunidades para que estos adquieran una visión crítica y activista en cuanto a la tecnología, se priorizan cursillos, seminarios y talleres para, en teoría, favorecer la competencia digital pero que, en realidad, se enfocan en cómo usar una herramienta (Escudero et al., 2018; Saura et al., 2021). Asimismo, la rapidez con la que se producen los cambios tecnológicos, y la consiguiente aparición y desaparición de herramientas, alimenta aún más la sensación de urgencia en cuanto a la formación tecnológica puntual, en detrimento de visiones más holísticas, reposadas y racionales que tengan en cuenta por igual a todos los integrantes del ecosistema del aprendizaje y las relaciones que se establecen entre ellos. Esta tendencia se agudizó durante la pandemia, cuando la coyuntura de la inmediatez obligó a hacer educación a pesar del virus. La tecnología en la educación en la pandemia fue una salida rápida, pero no una solución estable.

Otro aspecto llamativo dentro de este discurso centrado en las herramientas tecnológicas consiste en la idea, bastante generalizada desde hace décadas, de que la disrupción o los cambios en educación se van a producir como resultado de la introducción de nuevas tecnologías en el aula (Area y Adell, 2021; Cuban \& Jandrić, 2015; Sangrà Morer et al., 2021). No hay ninguna duda de que el hecho de realizar determinadas tareas utilizando herramientas tecnológicas puede tener un impacto en la forma en la que organizamos la actividad de docentes y discentes. Pero, si se parte exclusivamente de aquello que la tecnología ofrece o promete ofrecer, se corre el riesgo de reconducir los cambios en la educación hacia las metas que los creadores de esas herramientas han identificado como prioridades educativas y como problemas que necesitan ser resueltos (Castañeda \& Selwyn, 2018; Ideland, 2021; Selwyn et al., 2020; Teräs et al., 2020). Dado que gran parte de estas herramientas vienen de la mano de grandes empresas tecnológicas globales, conocidas con el acrónimo GAFAM —es decir, Google, Apple, Facebook, Amazon y Microsoft-, resulta necesario plantearse hasta qué punto su eduvisión es compatible con las características específicas de sistemas educativos diferentes o solo parte de un gran negocio. En otras palabras, ¿estamos haciendo innovación educativa partiendo de lo que queremos que aporte nuestro sistema educativo a la sociedad o estamos intentando modificar nuestro entorno educativo conforme al discurso y los modelos ofrecidos por las grandes empresas tecnológicas? En este contexto es importante cuestionarse qué ganan las empresas tecnológicas con la tecnologización de la educación y la datificación del aprendizaje (Saura et al. 2021, Tëras et al., 2021). De nuevo, estos temas son aún más relevantes a la luz de que la mayor parte de las tecnologías implementadas durante la educación digital de emergencia vinieron de la mano de grandes compañías tecnológicas de ámbito global. Estas empresas ni siquiera tuvieron en cuenta los condicionantes educativos de los países en los que desarrollaron dichas herramientas (Garcia \& Philip, 2021), por lo que es fácil deducir que su interés por proporcionar soluciones específicas a las necesidades de otros sistemas educativos fue prácticamente inexistente.

Asimismo, no hay que pasar por alto que, por mucho tiempo, la estrategia más empleada para convencer al profesorado reacio a la incorporación de la tecnología educativa consistía en resaltar el hecho de que esta no iba a sustituirlo, sino que simplemente le permitiría llevar a cabo su práctica docente de una manera eficiente, esto es, de forma más rápida y organizada. Pocas veces se invitaba al profesorado, y mucho menos al alumnado, a participar en el desarrollo de soluciones tecnológicas proporcionando ideas sobre cómo querían o necesitaban cambiar en el proceso de enseñanza/aprendizaje (Garcia \& Philip, 2021). Y en las ocasiones en las que esto ha sucedido se ha hecho, la inercia y las condiciones del mercado que rodea a la educación han impedido que se progrese en ese sentido o han reconducido el debate hacia soluciones que aumentan las ganancias de las empresas (Garcia \& Philip, 2021; Teräs et al., 2020; Williamson \& Hogan, 2020); un ejemplo de ello es la mercadotecnia editorial conocida como "facturación automática y acceso inclusivo". Se trata de un programa de las grandes editoriales estadounidenses de libros de texto para universidades creado en respuesta a las fundadas 
críticas contra el constante y gravoso aumento del precio de los libros de texto. Comercializada y promocionada como una solución para abaratar el precio de la educación superior, no siempre repercute, en realidad, en beneficio del alumnado, que se ve obligado a abonar el acceso a los libros digitales junto a la matrícula del curso sin recibir garantías en cuanto a la devolución de lo abonado si decide no quedarse en el curso, o sin poder revender el código de acceso cuando este termine (Williamson, 2019). En la educación pospandémica es necesario estar más vigilantes que nunca ante este tipo de soluciones implementadas sin la participación de todos los miembros del ecosistema educativo.

A la vista de todo lo anterior, si se pretende investigar las circunstancias tecnopedagógicas que pueden favorecer un acercamiento digital al proceso de enseñanza-aprendizaje, con ánimo de mejorarlo, se debe ir más allá de la descripción de la idoneidad de una herramienta específica para solventar o hacer más eficiente una tarea educativa concreta, y enfocarse en propuestas educativas con tecnología más integrales y participativas, entendidas estas como modelos educativos digitales, es decir, respuestas educativas concretas donde la tecnología se utiliza de forma sistemática, ética y crítica. Como cualquier otro modelo educativo, los modelos educativos digitales son constructos teóricos que necesitan ir refrendados por un adecuado proceso de investigación que permita verificar su validez y su replicabilidad, con las debidas adaptaciones, a contextos educativos diferentes de los que los hicieron surgir. A tal efecto, el concepto de ecosistema del aprendizaje se perfila como un marco de gran utilidad para el estudio de los elementos necesarios en cualquier modelo educativo digital y para investigar $-\mathrm{y}$ si es necesario, contrarrestar- los ajustes que se producen en el modelo cuando se introducen sin cuidado tecnologías foráneas al sistema.

\section{ECOSISTEMAS DE APRENDIZAJE: RESPUESTAS LOCALES A PRESIONES GLOBALES}

La noción de ecosistema de aprendizaje irrumpe en el campo educativo a finales del siglo XX y desde entonces ha sido aplicada a áreas muy diversas, como la formación empresarial (Chang \& Guetl, 2007), el análisis de la innovación educativa o la introducción de herramientas tecnológicas (Castañeda \& Adell, 2013; García Peñalvo, 2018; Martí et al., 2018; Zhao \& Frank, 2003). Aunque resulta sencillo entender por qué la metáfora del ecosistema —entendido este como un conjunto de elementos bióticos y abióticos que se relacionan entre sí en un espacio a lo largo del tiempo- puede ser útil en el campo de la educación, no es tan fácil llegar a un consenso acerca de cómo conceptualizar esta noción. Martí et al. (2018) recogen diversas propuestas realizadas por otros autores para definir el ecosistema educativo tecnológico. Asimismo, describen algunas de sus características, entre las que destacan la disponibilidad, la accesibilidad, la relevancia, la calidad, la interoperabilidad, la evolucionabilidad, la eficacia, la sostenibilidad, la escalabilidad y la gobernabilidad. Según Chang y Guetl (2007), el profesorado, el alumnado, la administración del centro escolar, las familias y el entorno social son los componentes vivos de los ecosistemas de aprendizaje. Los contenidos, el currículum, las herramientas y otros materiales para el aprendizaje constituyen los componentes abióticos. Sin embargo, para otros autores como Zhao y Frank (2003), la constante evolución de la tecnología como elemento que sustituye a los seres humanos en la realización de ciertas actividades le confiere a la tecnología educativa características más similares a las de los seres vivos, con lo cual pertenecería a los componentes bióticos del ecosistema de aprendizaje y no al grupo de los elementos no vivos del sistema, como podrían ser los libros o las condiciones físicas del centro educativo. Aparte de sus componentes, los ecosistemas de aprendizaje tienen límites y están sujetos a influencias tanto externas como externas. Por ejemplo, siguiendo a Zhao y Frank (2003), cada escuela constituye un ecosistema de aprendizaje que, por su parte, pertenece a ecosistemas mayores, como el distrito escolar, los cuales, a su vez, se engloban bajo el ecosistema educativo de la región y así sucesivamente. Dentro de cada ecosistema, cualquier cambio en uno de sus componentes afecta necesariamente al resto; lo mismo sucede cuando se introducen nuevos componentes en el ecosistema. Por lo tanto, los ecosistemas, para no desaparecer como tales, deben disponer de flexibilidad para adaptarse a los cambios, pero, al mismo tiempo, deben disponer de la suficiente estabilidad para evitar su extinción. 
La tensión entre la adaptabilidad y la continuidad se manifiesta en todos los niveles o capas de los que se compone el ecosistema, los cuales Gu et al. (2019) denominan en macro-, mesoy micronivel. En el nivel macro se encuentran todos los aspectos que rodean al ecosistema en cuestión, es decir, su capa más externa. Las dinámicas de cambio que se producen desde este nivel son determinadas, por ejemplo, por los gobiernos a través de leyes educativas o por la influencia de grupos de presión. Otros agentes menos evidentes, pero con un gran poder en el campo educativo, son las grandes editoriales y las empresas tecnológicas, como ya se ha mencionado más arriba. En el nivel meso encontramos todas las dinámicas que se producen dentro del ecosistema escolar. En el caso de la tecnología, agentes como los especialistas en diseño instruccional locales o los encargados de adquirir nuevas herramientas desempeñan un papel fundamental en este nivel. Por último, en el nivel micro, y también en cuanto a la introducción de la tecnología, Gu et al. (2019) describen las dinámicas que existen en la adopción de herramientas concretas dentro de la práctica docente individual. En el caso de la educación digital de emergencia durante la pandemia, la tecnología se introdujo en las tres capas del ecosistema educativo, provocando cambios de intensidad en el resto de sus componentes sin que se pudieran implementar mecanismos para suavizar las consecuencias menos beneficiosas. Pero ahora ha llegado el momento de recapacitar.

Está claro que la complejidad de las características de los componentes de cualquier ecosistema de aprendizaje y de las relaciones y dinámicas que se establecen entre ellos a lo largo del tiempo dificultan sobremanera establecer modelos educativos digitales que puedan ser susceptibles de ser aplicados universalmente a cualquier contexto educativo. Averiguar cuáles son las condiciones que determinan la idoneidad de un determinado modelo educativo digital es un proceso complejo que comienza con el estudio exhaustivo de las características específicas del ecosistema en el que se vaya a implementar dicho modelo educativo digital. El proceso se ha complicado aún más tras la pandemia ya que, como se apunta en Teräs et al., (2020, p. 668), "debido a que la tecnología se construye socialmente (Selwyn, 2010), las decisiones tomadas durante la pandemia en cuanto a las herramientas tecnológicas y su papel en la organización pedagógica, tendrán un impacto tanto en las experiencias futuras de enseñanza y aprendizaje en el nivel micro como en las relaciones organizacionales en el nivel meso y en la sociedad a nivel macro".

Entonces, ¿cómo se puede identificar el impacto de la educación digital de emergencia y aprovecharlo para desarrollar modelos digitales para el futuro a la vista de la complejidad y mutabilidad de las interacciones que se producen dentro de los ecosistemas de aprendizaje? Describir aquí con detalle todas las variables que se deben considerar a tal efecto excedería los límites de este artículo, que solo pretende tener un carácter introductorio y, como tal, estimular una forma holística de pensar la educación, con o sin pandemia, considerado todos aquellos factores que a menudo no suelen formar parte de la planificación, la organización, la implementación y la evaluación de la digitalización del proceso de enseñanza/aprendizaje en ninguna de las capas del ecosistema. Entre ellos, se podrían mencionar, por lo evidentes que son, la influencia que ejercen las herramientas tecnológicas en la estructuración, secuenciación y presentación de contenidos y en el diseño de ejercicios y actividades de evaluación en entornos online; o la extracción, almacenamiento y análisis de todo lo datificable en el proceso de enseñanza/aprendizaje sin que las personas que han generado esos datos puedan oponerse a su monetización por parte de las empresas tecnológicas que les han vendido la herramienta (Saura et al., 2021). Hay que recordar de nuevo que el elemento tecnológico se introduce en el ecosistema de aprendizaje de forma no consensuada y que, cada vez con más frecuencia, está compuesto por recursos desarrollados para contextos educativos indefinidos y homogeneizadores. Las soluciones tecnológicas locales son escasas y la posibilidad de adaptar las soluciones globalizadoras a contextos más locales se limitan, casi de forma exclusiva, a aspectos lingüísticos (por ejemplo, la disponibilidad de la herramienta en varios idiomas). En realidad, no interesa adaptar estas herramientas tecnológicas globales porque cuanto más adaptadas estén al entorno, menor será la posibilidad de extraer datos homogéneos y de establecer relaciones rentistas de datos entre varias plataformas (Saura et al., 2021). Es el caso de la plataformización de la enseñanza, un fenómeno ya existente antes de la pandemia pero que se ha incrementado astronómicamente durante esta (Rivas, 2021; Saura et al., 2021). La plataformización, impulsada por las GAFAM, se observa en todos los ámbitos de la sociedad digital y ha llamado la atención de todos los gobiernos a nivel mundial (Van Dijck, 2021). En el 
campo de la educación, mientras que países como China y Corea del Sur han optado por un control gubernamental de las plataformas educativas, en Estados Unidos las plataformas de aprendizaje, "impulsadas por los datos y gobernadas por sus algoritmos" (Van Dijck, 2021, p. 2804) están en manos de las grandes empresas de EdTech. La Unión Europea, sin ningún jugador de peso en esta lucha por el mercado del aprendizaje, se ha intentado posicionar, sobre todo, como agente de cambio gubernamental en la sociedad digital (Van Dijck, 2021, p. 2803). La plataformización de la sociedad y de la enseñanza no se puede frenar, pero lo que sí se debe hacer es exigir mayor responsabilidad a las grandes empresas de EdTech y concentrar las iniciativas de formación tecnológica no tanto en el uso de una herramienta para realizar una tarea, sino en el estudio de las implicaciones que las decisiones que se toman cuando se diseña cierta herramienta tecnológica tienen sobre los demás elementos del ecosistema de aprendizaje.

En la pandemia se vivió quizá la experiencia más integral de plataformizacion de la educación hasta ahora conocida. Durante la educación digital de emergencia, la forma más rápida de continuar la educación fue la introducción de plataformas ofrecidas por alguna de las GAFAM, como Google Classroom o Microsoft Teams, soluciones que se podría etiquetar de globales y que, en la premura pandémica, se introdujeron por ensayo y error y sin ningún control, como si de una plaga se tratara, en los ecosistemas de aprendizaje locales. No se pudo hacer de otra manera. Pero ahora es el momento de pararse a reflexionar y de la mano de trabajos tan sobresalientes como los de Rivas (2021), Saura et al. (2021), Selwyn et al. (2021) y Teräs et al. (2021), seguir construyendo una sólida base de estudios que hagan frente a las tendencias globalizadoras de las grandes empresas tecnológicas, que descolonicen el campo de la EdTech y que sepan aprovechar mejor la potencialidad de la tecnología actual para crear verdaderas experiencias de aprendizaje participativo, ético y transformador. Hay muchos factores en contra, como los discursos imperantes sobre tecnología (Castañeda, 2021) o la aceptación por parte de los más jóvenes de las condiciones que impone la datificación y el colonialismo de datos como la única forma de acceder a los beneficios que ofrecen las GAFAM y otras empresas de servicios digitales (Fernández-Rovira \& Giraldo-Luque, 2021). Sin embargo, dado lo mucho que está en juego, se hace necesario un enfoque que permita romper con el solucionismo tecnológico y aporte una forma de entender y estudiar la tecnología educativa como una parte más del ecosistema de aprendizaje, con sus influencias negativas y positivas para el resto de los elementos que lo configuran.

\section{EN ESTE MONOGRÁFICO}

El enfoque reflexivo, holístico y pedagógico que subyace a este artículo y a todos los incluidos en este volumen permite desvincular la tecnología digital de los calificativos con los que con más frecuencia, y de forma errónea, se asocia: la tecnología como redentora de todos los males de la educación y la tecnología como la causante de muchos de sus problemas. La tecnología no es ni una cosa ni la otra: es simplemente una pieza más que debe estar articulada con la inmensa complejidad de aquello que consideramos la educación. La tecnología sin pedagogía no funciona (Suárez-Guerrero et al., 2016), por ello es necesario pensar en los modelos educativos que brinden un enfoque integrador a la tecnología digital y que sean conscientes de las dinámicas que se producen dentro del ecosistema, sobre todo tras su rápida y apenas planificada introducción durante la pandemia. Integrar la tecnología en el modelo educativo considerando a todos los agentes externos y a los componentes del ecosistema brinda esa coherencia básica para, entre otros aspectos, alejarse del tecnocentrismo que distrae la esencia de la acción educativa. La educación digital no consiste en ceder a las presiones que se producen en los niveles macro, meso o micro, sino elaborar un conjunto integral de experiencias en las que ni la tecnología ni sus promotores se conviertan en depredadores del ecosistema. Por eso es tan importante, tanto a nivel micro- como macroeducativo, con o sin pandemia, "orquestar" — como señala Dillenbourg (2013) — la tecnología al diseño de las actividades educativas. Y esta es la clave de los enfoques basado en modelos de aprendizaje digital, como sostiene Guàrdia (2020, p. 47) que tienen "por objetivo que se tome consciencia de que no es más importante el diseño de los contenidos que el diseño metodológico o la tecnología que se utilizará, sino la capacidad de integrarlo todo al servicio de las necesidades de aprendizaje y de acuerdo con el perfil del alumnado". Solo considerando que la tecnología es un elemento más del ecosistema de aprendizaje, la tecnología podrá ser parte relevante de la idea educativa. Pero, como todo 
modelo es un constructo teórico y, como tal, necesita contraste; a tal efecto, este monográfico presenta cuatro de estos desarrollos validados en la práctica y sometidos a revisión.

En "Educación online durante la COVID-19: problemáticas afrontadas por los docentes", Elena Vela parte de una de las consecuencias negativas que con mayor frecuencia se cita tanto en la bibliografía general sobre la introducción de las tecnologías educativas en el currículum como en los estudios realizados hasta la fecha sobre la pandemia de la COVID-19. Se trata de la brecha educativa y de cómo la implementación de modelos educativos basados en tecnología, en lugar de mejorar el acceso a la educación entre las poblaciones más vulnerables, refuerza que los que más recursos poseen también sean los que más facilidades tienen para obtener éxito en su educación. Para estudiar con mayor profundidad el impacto de la introducción de las TIC durante la pandemia entre los grupos más vulnerables, Elena Vela se centra en los cambios realizados por los docentes de los centros del Plan de Compensación Educativa de la Junta de Andalucía (España), en la valoración que el profesorado hizo sobre la coordinación y organización docente y en las dificultades que tuvieron para llevar a cabo su labor educativa durante la pandemia. Los resultados de su investigación dejan patente que la falta de formación digital de docentes, discentes y familias, además del limitado acceso a la tecnología, afectó negativamente al desarrollo educativo del alumnado. Estas son variables críticas en la concepción de una educación con tecnología.

Jorge Antonio Balladares-Burgos, en "Percepciones en torno a una educación remota y a una educación híbrida universitaria durante la pandemia de la COVID-19: estudio de caso", describe un estudio de caso realizado en una universidad de Ecuador. En él se analiza la adaptación del diseño curricular del programa de posgrado de Especialización Superior en Educación y TIC de lo presencial a la educación remota mediada con la tecnología, prestando especial atención a la opinión de los estudiantes que realizaron este programa durante la pandemia. Además, el artículo identifica una serie de aspectos relevantes en el modelo analizado: la temporalización de la enseñanza y el aprendizaje a través de las clases sincrónicas mediadas por videoconferencias, la utilización de entornos asincrónicos para realizar crear materiales y diseñar actividades autónomas, prácticas y colaborativas y la necesidad de que los centros universitarios garanticen la conectividad para evitar que determinados segmentos de la población se vean privados de la posibilidad de acceder a una enseñanza de calidad. El artículo termina con algunas recomendaciones para la enseñanza universitaria pospandemia que desee retomar la presencialidad a través de una educación híbrida. En general, el trabajo deja evidencia que la migración de lo presencial a lo virtual ni es una tarea mecánica ni está exenta de altibajos.

En "Herramientas digitales aplicadas en el escenario de la inclusión educativa en la época de la COVID-19", Estela Isequilla Alarcón y María Martín Delgado nos presentan un estudio realizado con una muestra de alumnado y profesorado de la provincia española de Málaga, mediante el cual se pretendía conocer qué herramientas utilizaron los dos grupos de informantes para las clases, para las tutorías y para compartir información durante la pandemia, además de preguntar qué herramientas nuevas se aprendieron a causa de la educación digital de emergencia provocada por la covid-19. Además, resalta la necesidad de atender a las necesidades socio-emotivas del alumnado, sobre todo en tiempos de situaciones de aislamiento y falta de contacto social, como la que se vivió durante el estado de alarma de 2020. Este trabajo aporta, además de las consabidas herramientas básicas para el diseño de modelos, un llamado a la necesidad de atender la dimensión emocional no solo en situaciones de pandemia, sino en la propia educación a distancia, que no tiene que ser distante en lo afectivo.

Por su parte, Belén Suárez Lantarón y Nuria García-Perales, en "Covid-19: La brecha (digital) educativa a través de la prensa", buscan poner en evidencia la necesidad de encarar el concepto de "brecha digital" en los escolares como variable crítica en la concepción de la educabilidad digital en la pandemia. Para ello exploran, desde un enfoque cualitativo apoyado en el análisis de contenido, la prensa española generalista, tanto de ámbito nacional como autonómico, durante el primer mes de confinamiento. La prensa incide en el desequilibrio y la desigualdad social respecto al acceso a la información y las dificultades para acceder a la solución educativa online, identificando tres causas explicativas con que el ámbito periodístico explica la brecha digital en la escuela: ligadas a la familia (precariedad laboral o diferencias socioculturales, por ejemplo), en torno a los recursos (sin conexión o limitaciones del equipo, por ejemplo) y respecto al entorno (rural o urbano). El trabajo pone de manifiesto que la prensa 
generalista ha incrementado la visibilidad de una desigualdad digital que hay gestionar para que la escuela digital de emergencia no prescinda de los ya excluidos.

Estos trabajos reflejan, con mayor o menor profundidad, el proceso que siguen las ideas y la reflexión en torno a los modelos educativos que sostienen el uso de la tecnología y cómo esta ha influido al resto del ecosistema de aprendizaje. Son parte del proceso de diálogo que más temprano que tarde deben de formar parte de la esa noción de escuela pos-COVID-19 que, según Jandrić (2020), va a seguir viviendo y reflexionando porque es un hito en la teoría y la práctica educativa. El objetivo no es la perfección, sino seguir aprendiendo para hacer perfectible la compleja tarea que es educar.

\section{REFERENCIAS BIBLIOGRÁFICAS}

Area, M., \& Adell, J. (2021). Tecnologías Digitales y Cambio Educativo. Una Aproximación Crítica. REICE. Revista Iberoamericana sobre Calidad, Eficacia y Cambio en Educación, 19(4), 83-96. https://doi.org/10.15366/reice2021.19.4.005

Burgess, S. (2014). Centre-Periphery Relations in the Spanish Context: Temporal and CrossDisciplinary Variation. En: K. Bennett K. (Ed.), The Semiperiphery of Academic Writing (pp. 93-104). Palgrave Macmillan.

Castañeda, L. (2021). Trazabilidad de los discursos sobre tecnología educativa: los caminos de la influencia. Revista Interuniversitaria de Investigación en Tecnología Educativa, 10, 1-8. https://doi.org/10.6018/riite.480011

Castañeda, L., \& Adell, J. (Eds.). (2013). Entornos Personales de Aprendizaje: claves para el ecosistema educativo en red. Marfil.

Castañeda, L., \& Selwyn, N. (2018). More Than Tools? Making Sense of the Ongoing Digitalizations of Higher Education. International Journal of Educational Technology in Higher Education 15: 1-10.

Cuban, L., \& Jandrić, P. (2015). The dubious promise of educational technologies: Historical patterns and future challenges. E-Learning and Digital Media, 12(3-4), 425-439. https://doi.org/10.1177/2042753015579978

Dillenbourg, P. (2013). Design for classroom orchestration. Computers \& Education, 69, 485492.

Escudero, J. M., Martínez, B., \& Nieto, J. M. (2018). Las TIC en la formación continua del profesorado en el contexto español. Revista de Educación, 382, 57-80. http://doi.org/10.4438/1988-592X-RE-2018-382-392

Fernández-Rovira, C., \& Giraldo-Luque, S. (2021). Evolution of the Digital Attention Market in the Pandemic: A Comparative Study of Young Spanish University Students (2019-2021). Sustainability, 13, 11837. https://doi.org/10.3390/su132111837

Garcia, A., \& Philip, N. T. (2021). Digital Platforms aren't Mere Tools - They're Complex Environments. Phi Delta Kappan, 102(6), 14-19.

García-Peñalvo, F. J. (2018). Ecosistemas tecnológicos universitarios. En J. Gómez (Ed.), UNIVERSITIC 2017. Análisis de las TIC en las Universidades Españolas (pp. 164-170). Crue Universidades Españolas.

Fernández Enguita, M. (13 de marzo de 2020). Una pandemia imprevisible ha traído la brecha previsible. Cuaderno de Campo. https://blog.enguita.info/2020/03/una-pandemiaimprevisible-ha-traido-la.html

Gu, X., Crook, C., \& Spector, M. (2019). Facilitating innovation with technology: Key actors in educational ecosystems. British Journal of Educational Technology, 50(3), 1118-1124 doi:10.1111/bjet.12786

Guàrdia, L. (2020). Diseño de cursos online, en A. Sangrà (Coord). Decálogo para la mejora de la docencia online, 45-62. Editorial UOC. http://hdl.handle.net/10609/122307 
Hodges, C., Moore, S., Lockee, B., Trust, T., \& Bond, A. (March 27, 2020). The difference between emergency remote teaching and online learning. Educause Review. https://bit.ly/3b0Nzx7

Hyland, K. (2012). Academic Publishing. Issues and Challenges in the Construction of Knowledge. Oxford University Press.

Ideland, M. (2021). Google and the end of the teacher? How a figuration of the teacher is produced through an ed-tech discourse. Learning, Media and Technology, 46(1), 33-46, DOI: 10.1080/17439884.2020.1809452

Jandrić, P. (2020). The Day After Covid-19. Postdigit Sci Educ 2, 531-537. https://doi.org/10.1007/s42438-020-00195-4

Law, N. \& Liang, L. (2019). Socio-technical co-evolution of an e-learning innovation network. British Journal of Educational Technology, 50(3), 1340-1353. https://doi.org/10.1111/bjet.12768

Ornellas A., \& Sancho, J. (2015). Three Decades of Digital ICT in Education: Deconstructing Myths and Highlighting Realities. En: M. K. Harmes, M. Huijser \& P. Danaher (Eds.) Myths in Education, Learning and Teaching, (pp. 135-150). Palgrave Macmillan. https://doi.org/10.1057/9781137476982 8

Organización de las Naciones Unidas [ONU] (2020). Informe de políticas: la educación durante la COVID-19 y después de ella.https://bit.ly/3pG1INR

Pulido-Montes, C., \& Ancheta-Arrabal, A. (2021). La educación remota tras el cierre de escuelas como respuesta internacional a la Covid-19. Revista Prisma Social, 34, 236-266. https://revistaprismasocial.es/article/view/4217

Sangrá Morer, A., Raffaghelli, J. E., González Sanmamed, M., \& Muñoz Carril, P. C. (2021). Desarrollo profesional de maestros de primaria desde la óptica de las ecologías de aprendizaje: nuevas formas de actualizarse en tiempos inciertos. Publicaciones, 51(3), 2170. https://doi.org/10.30827/publicaciones.v51i3.20790

Saura, G., Díez Gutiérrez, E. J., \& Rivera Vargas, P. (2021). Innovación Tecno-Educativa "Google". Plataformas Digitales, Datos y Formación Docente. REICE. Revista Iberoamericana sobre Calidad, Eficacia y Cambio en Educación, 19(4). https://doi.org/10.15366/reice2021.19.4.007.

Selwyn, N. (2010). Looking beyond learning: Notes towards the critical study of educational technology. Journal of Computer Assisted Learning, 26(1), 65-73. https://doi.org/10.1111/j.1365-2729.2009.00338.x.

Selwyn, N., Hillman, T., Eynon, R., Ferreira, G., Knox, J., Macgilchrist, F., \& Sancho-Gil, J. M. (2020). What's next for Ed-Tech? Critical hopes and concerns for the 2020s. Learning, Media and Technology, 45(1), 1-6. https://doi.org/10.1080/17439884.2020.1694945.

Suárez-Guerrero, C. (2014). Pedagogía red. Cuadernos de pedagogía, 449, 76-80. Disponible en: http://bit.ly/1nfogNf

Suárez-Guerrero, C., Lloret-Catalá, C., \& Mengual-Andrés, S. (2016). Teachers' perceptions of the digital transformation of the classroom through the use of tablets: A study in Spain. Comunicar, 49, 81-89. https://doi.org/10.3916/C49-2016-08

Teräs, M., Suoranta, J., Teräs, H., \& Curcher, M. (2020). Post-Covid-19 Education and Education Technology 'Solutionism': a Seller's Market. Postdigital Science and Education, 2, 863-878. https://doi.org/10.1007/s42438-020-00164-x

Rivas, A. (2021). The Platformization of Education: A framework to Map the New Directions of Hybrid Education Systems. In-Progress Reflection, 46. IBE-UNESCO.

Van Dijck, J. (2021). Seeing the forest for the trees: Visualizing platformization and its governance. New Media \& Society, 23(9) 2801-2819 https://journals.sagepub.com/doi/10.1177/1461444820940293 
Williamson, J. (31 de julio de 2019). What you should ask about inclusive access programs to ensure your students have positive experiences now and their interests are protected in the future. $\quad$ https://openstax.org/blog/if-inclusive-access-horizon-ask-yourself-these-ninequestions

Williamson, B., Rebecca, E., \& Potter, J. (2020). Pandemic politics, pedagogies and practices: digital technologies and distance education during the coronavirus emergency. Learning, Media and Technology, 45(2), 107-114. https://doi.org/10.1080/17439884.2020.1761641

Williamson, B., \& Hogan, A. (2020). La comercialización y la privatización en y de la educación en el contexto de la COVID-19. Internacional de la educación. https://ei-ieal.org/sites/default/files/docs/20200708 102224 la comercializacion y la privatizacion en y de la educacion en el contexto de la covid-19.pdf

World Bank (2020). The Covid-19 pandemic: shocks to education and policy responses. World Bank.https://bit.ly/3grkejE

Zhao, Y., \& Frank, K. A. (2003). Factors Affecting Technology Uses in Schools: An Ecological Perspective. American Educational Research Journal, 40(4): 807-840.

\section{INFORMACIÓN SOBRE LOS AUTORES}

\section{Esperanza Román Mendoza \\ George Mason University}

Doctora en Lengua Española y Lingüística General, es Catedrática de Lingüística Española en George Mason University (EE. UU.). Sus áreas de especialización son el e-learning, la educación a distancia y la enseñanza del español a hispanohablantes en Estados Unidos desde la perspectiva de la pedagogía crítica. Ha publicado libros y artículos sobre diferentes aspectos de la incorporación de la tecnología en el aprendizaje, como la monografía Aprender a aprender en la era digital. Tecnopedagogía crítica para la enseñanza de español LE/L2 (Routledge 2018), además de materiales didácticos digitales tanto de acceso gratuito como comerciales. Forma parte de grupos de investigación de la Universidad de Salamanca, la Universitat de València y la Universidad Complutense de Madrid, entre otros. Más información sobre ella en http://mcl.gmu.edu/people/eromanme.

\section{Cristóbal Suárez-Guerrero \\ Universitat de València}

Profesor Titular del Departamento de Didáctica y Organización Escolar de la Universitat de València. Doctor en Educación en procesos de formación en espacios virtuales por la Universidad de Salamanca. Es miembro del grupo de investigación en pedagogías culturales (CREARI) de la Universitat de València y sus líneas de trabajo son: pedagogía digital, innovación didáctica con tecnología, humanidades y cultura digital en educación, enfoque sociocultural y aprendizaje cooperativo y aprendizaje abierto en red. Más información sobre él en su blog: http://educacionvirtualidad.blogspot.com/ y en su cuenta de Twitter: https://twitter.com/cristobalsuarez

\section{(9) $(1)(20$}

Los textos publicados en esta revista están sujetos a una licencia de Reconocimiento 4.0 España de Creative Commons. Puede copiarlos, distribuirlos, comunicarlos públicamente y hacer obras derivadas siempre que reconozca los créditos de las obras (autoría, nombre de la revista, institución editora) de la manera especificada por los autores o por la revista. La licencia completa se puede consultar en:Licencia Creative Commons Atribución-NoComercial-Compartir por igual 4.0 Internacional. 